ARTICLE

DOI: $10.1038 / s 41467-017-00406-w$

\title{
Regulation of RIPK1 activation by TAK1-mediated phosphorylation dictates apoptosis and necroptosis
}

Jiefei Geng ${ }^{1}$, Yasushi Ito', Linyu Shi ${ }^{2}$, Palak Amin (1) 1, Jiachen $\mathrm{Chu}^{1}$, Amanda Tomie Ouchida', Adnan Kasim Mookhtiar', Heng Zhao ${ }^{1}$, Daichao Xu ${ }^{1}$ Bing Shann ${ }^{2}$, Ayaz Najafov (1) ${ }^{1}$, Guangping Gao ${ }^{3}$, Shizuo Akira ${ }^{4} \&$ Junying Yuan ${ }^{1,2}$

Stimulation of TNFR1 by TNF $\alpha$ can promote three distinct alternative mechanisms of cell death: necroptosis, RIPK1-independent and -dependent apoptosis. How cells decide which way to die is unclear. Here, we report that TNF $\alpha$-induced phosphorylation of RIPK1 in the intermediate domain by TAK1 plays a key role in regulating this critical decision. Using phospho-Ser321 as a marker, we show that the transient phosphorylation of RIPK1 intermediate domain induced by TNF $\alpha$ leads to RIPK1-independent apoptosis when NF-KB activation is inhibited by cycloheximide. On the other hand, blocking Ser321 phosphorylation promotes RIPK1 activation and its interaction with FADD to mediate RIPK1-dependent apoptosis (RDA). Finally, sustained phosphorylation of RIPK1 intermediate domain at multiple sites by TAK1 promotes its interaction with RIPK3 and necroptosis. Thus, absent, transient and sustained levels of TAK1-mediated RIPK1 phosphorylation may represent distinct states in TNF-RSC to dictate the activation of three alternative cell death mechanisms, RDA, RIPK1independent apoptosis and necroptosis.

\footnotetext{
${ }^{1}$ Department of Cell Biology, Harvard Medical School, 240 Longwood Ave, Boston, MA 02115, USA. ${ }^{2}$ Interdisciplinary Research Center on Biology and Chemistry, Shanghai Institute of Organic Chemistry, Chinese Academy of Sciences, 26 Qiuyue Rd, PuDong District, Shanghai 201210, China. ${ }^{3}$ Horae Gene Therapy Center and Vector Core, and Department of Physiological Systems, University of Massachusetts Medical School, 368 Plantation Street, AS6-2049, Worcester, MA 01605, USA. ${ }^{4}$ Laboratory of Host Defense, WPI Immunology Frontier Research Center (IFReC), Osaka University, 3-1 Yamadaoka, Suita, Osaka 565-0871, Japan. Correspondence and requests for materials should be addressed to J.Y. (email: jyuan@hms.harvard.edu)
} 
R IPK1, a member of the receptor-interacting protein (RIP) serine-threonine kinase family, has emerged as a key upstream regulator that controls multiple downstream signaling pathways of TNFR $1^{1,2}$. Within minutes after cells stimulated by TNF $\alpha$, RIPK1 is recruited into the TNFR1 signaling complex (TNF-RSC, also called complex I) together with signaling molecules such as TRADD, TRAF2 and cIAP1/2 to decide if a cell and ultimately, an organism, may live or die through apoptosis or necroptosis. Apoptosis may be mediated by binding of RIPK1, independent of its kinase activity, with FADD, an adaptor protein for caspase- 8 , which in turn promotes the activation of caspase- 8 and executes apoptosis by triggering mitochondrial damage and the cleavage of downstream caspases such as caspase-3. Under apoptotic deficient conditions, RIPK1 may be activated to promote necroptosis by interacting with RIPK3 which in turn promotes the phosphorylation of MLKL to mediate the execution of necroptosis.

Ubiquitination of RIPK1 by cIAP1/2 in TNF-RSC is involved in mediating the activation of $\mathrm{NF}-\kappa \mathrm{B}$ by recruiting $\mathrm{TAB} 1 / 2$ to promote the activation of the TAK1 (transforming growth factor$\beta$-activated kinase 1 , also called MAP3K7) ${ }^{3}$. Activated TAK1 mediates the phosphorylation of IKK $\beta$ to promote the formation of the IKK complex consisting of IKK $\alpha / \beta / \gamma(\mathrm{NEMO})^{4}$. Although the best characterized function of TAK1 and the IKK complex including NEMO is to mediate the activation of NF- $\kappa B$ pathway, recent studies have unveiled that deficiencies in TAK1, NEMO, IKK $\alpha / \beta$ or the loss of cIAP $1 / 2$ can sensitize cells to RIPK1dependent apoptosis (RDA) independently of their roles in NF- $\kappa \mathrm{B}$ activation ${ }^{5,6}$. On the other hand, in cells deficient for A20, an important ubiquitin-editing enzyme for RIPK1, or TAB2, which regulates the activation of TAK1, RIPK1 may be activated to interact with RIPK3 to mediate necroptosis ${ }^{7,} 8$. It is not clear, however, how activated RIPK1 might be directed to mediate two alternative modes of cell death, RDA or necroptosis, that both occur in a RIPK1 kinase-dependent manner.

RIPK1 contains an N-terminal kinase domain, an intermediate domain and a C-terminal death domain ${ }^{1}$. The kinase activity of RIPK1 may be activated upon stimulation of TNFR1 by TNF $\alpha$ under selective conditions, which leads to multiple deleterious consequences including cell death and inflammation. Inhibition of RIPK1 kinase activity using improved necrostatin-1 (R-7-ClO-Nec-1, Nec-1s), a highly specific small molecule inhibitor of RIPK1, and the use of RIPK1 kinase-dead mutant mice, have shown efficacy in a wide range of animal models of human diseases $^{9-11}$. Small molecule inhibitors of RIPK1 are under clinical and preclinical development targeting human diseases. However, the molecular mechanism that controls the activation of RIPK1 kinase activity remains unclear.

Here we show that the intermediate domain of RIPK1 is phosphorylated transiently by TAK1 upon TNF $\alpha$ stimulation in wild-type (WT) cells in vitro and in vivo. While Ser321 (S321) phosphorylation of RIPK1 by TAK1 has no effect on the NF- $\kappa$ B activation, the loss of $\mathrm{S} 321$ phosphorylation promotes the binding of RIPK1 to FADD and RDA. On the other hand, the sustained TAK1-mediated phosphorylation of RIPK1 in multiple sites of the intermediate domain including S321 promotes its interaction

a

310

320

330

Mus musculus LKKEYPं DSPVLORM F L LHDCV P LP PSRSNS Rattus norvegicus LKK EYPS OSPV LKRM F S LOHDCV P LP PSRSNS Homo sapiens LKKEYS NENAVVKRMOS LOLDCVAVPSSRSNS Bos taurus LKKEFPGONEIVKRMKS LOIDCVAIAPSRSNS $\triangle$

b

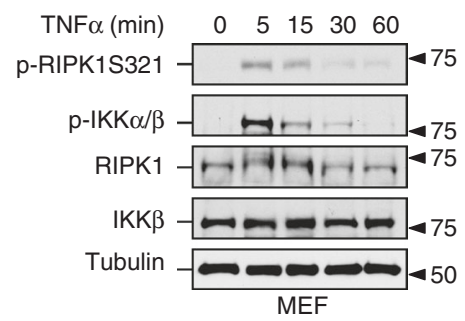

d

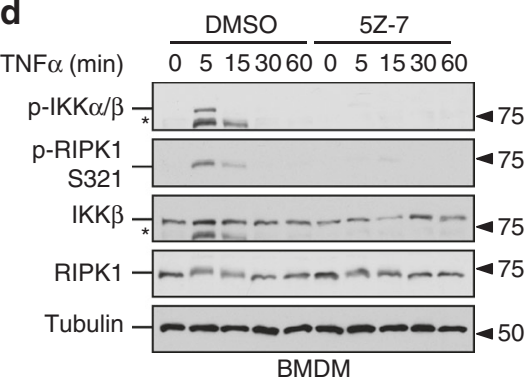

C

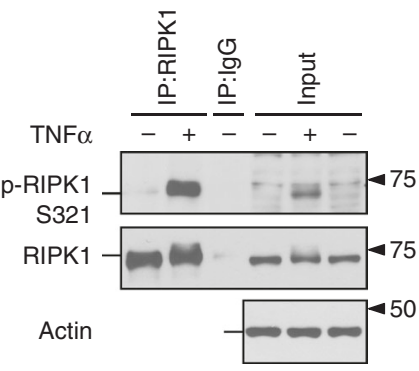

e

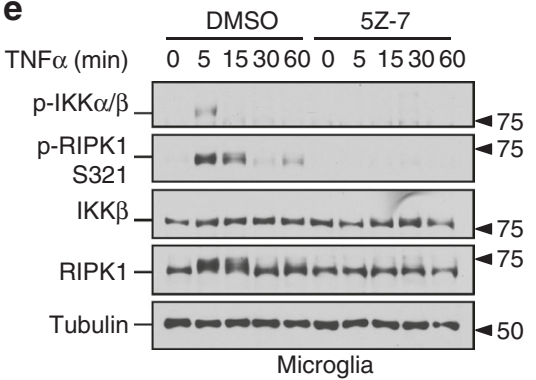

Fig. 1 TNF $\alpha$ induces RIPK1 phosphorylation at S321. a Alignment of amino acid sequences in the relevant part of RIPK1 intermediate domain from indicated mammalian species. S321, S332, S334 and S336 as marked by arrowheads are highly evolutionarily conserved. b S321 of RIPK1 is transiently phosphorylated after TNF $\alpha$ treatment. MEF cells were treated with TNF $\alpha(10 \mathrm{ng} / \mathrm{ml})$ and the samples were collected at indicted time-points. The cell lysates were analyzed by western blot. c Anti-p-S321-RIPK1 antibody specifically recognizes RIPK1 S321 phosphorylation. L929 cells were treated with TNF $\alpha$ $(20 \mathrm{ng} / \mathrm{ml})$ for $5 \mathrm{~min}$. The cell lysates were subjected to immunoprecipitation by either RIPK1 antibody or lgG. Both lysates and IP samples were examined by western blot using anti-p-S321-RIPK1 and RIPK1 total antibodies. d, e TNF $\alpha$ induces RIPK1 S321 phosphorylation in murine primary BMDM and microglia cells. BMDM d and primary microglia e were pre-incubated with DMSO or TAK1 inhibitor, $5 Z-7(0.5 \mu \mathrm{M})$ for $30 \mathrm{~min}$ and treated with TNF $\alpha(10 \mathrm{ng} / \mathrm{ml})$. Samples were collected at indicated time-points. ${ }^{*}$, nonspecific bands 
a

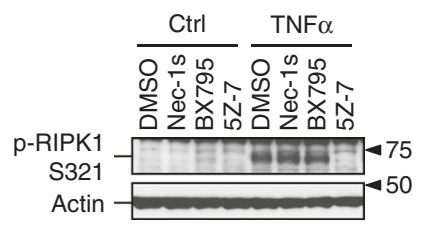

BV-2

C

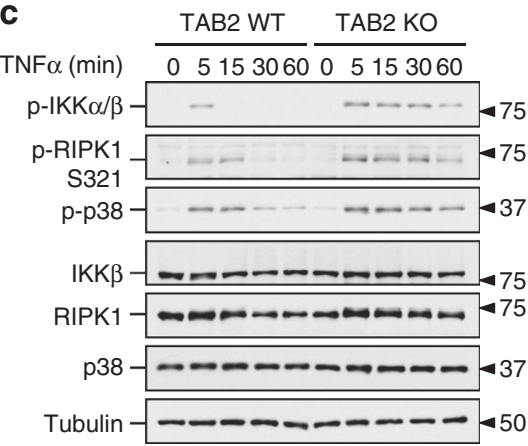

b

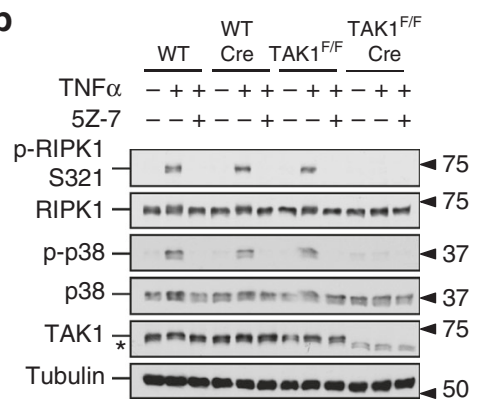

d

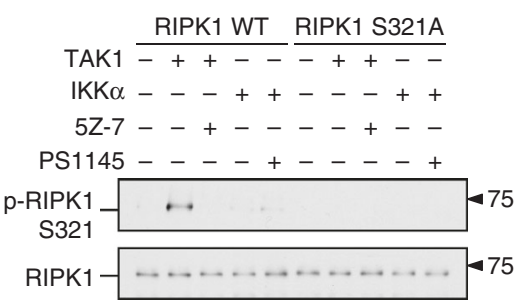

e

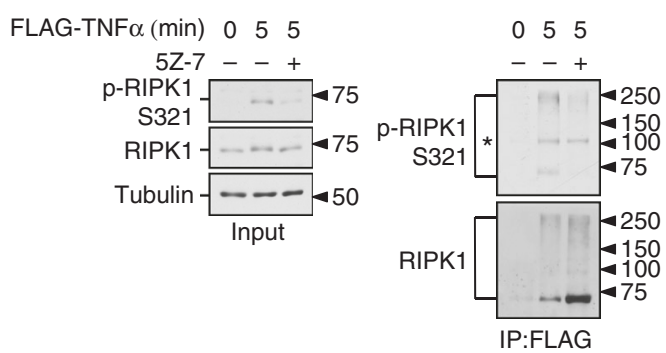

Fig. 2 TAK1 phosphorylates RIPK1 at S321. a TNF $\alpha$-induced RIPK1 S321 phosphorylation is inhibited by TAK1 inhibitor. BV-2 cells were treated by TNF $\alpha$ (20 $\mathrm{ng} / \mathrm{ml})$ together with DMSO, Nec-1s $(10 \mu \mathrm{M})$, TBK1 inhibitor, BX795 $(1 \mu \mathrm{M})$ and 5Z-7 (0.5 $\mu \mathrm{M})$. b TAK1 is required for RIPK1 S321 phosphorylation. WT and $T A K 1^{\mathrm{F} / F}$ MEFs with or without Cre expression were treated with TNF $\alpha(10 \mathrm{ng} / \mathrm{ml})$ and $5 Z-7(0.5 \mu \mathrm{M})$ for 15 min and examined by indicated antibodies. c Hyperphosphorylation of RIPK1 S321 in TAB2 KO MEF. TAB2 WT and KO MEFs were treated with TNF $\alpha$ (10 ng/ml) and the samples were collected at indicated time-points. d FLAG-RIPK1 WT and S321A were expressed and purified from 293T cells and incubated with recombinant TAK1-TAB1 or GST-IKK $\alpha$ or their inhibitors, 5Z-7 or PS1145, respectively. The products were analyzed by western blot using indicated antibodies. e RIPK1 S321 phosphorylation in TNF-RSC. MEF cells were treated with FLAG-TNF $\alpha(50 \mathrm{ng} / \mathrm{ml})$ for $5 \mathrm{~min}$ and TNF-RSC was purified by anti-FLAG immunoprecipitation. TNF-RSC immunocomplexes were analyzed by western blot using indicated antibodies. ${ }^{\star}$, nonspecific bands

with RIPK3 to mediate necroptosis. Our results elucidate the molecular mechanism of interaction between TAK1 and RIPK1, two critical mediators in the TNF $\alpha$ signaling pathway, distinct from their roles in the activation of the NF- $\kappa \mathrm{B}$ pathway, and the mechanism by which the levels of RIPK1 phosphorylation control the cellular choices for alternative cell death mechanisms.

\section{Results}

Transient RIPK1 S321 phosphorylation upon TNFo stimulation. S321 of RIPK1 was found to be phosphorylated in the kidney, lung and spleen tissues of mice under normal conditions in a global phosphoproteomic study and when expressed in 293 T cells $^{11}, 12$. S321 site is evolutionarily conserved in RIPK1 proteins from species including mouse, human, rat and cattle (Fig. 1a). S321 is located in a conserved sequence RMFSLQHDCV in murine RIPK1, or RMQSLQLDCV in human RIPK 1 . The +1 residue of this peptide is a 'Leu', which is also found in +1 residue of S177 in IKK $\beta$ known to be phosphorylated by TAK $1^{13}$.

To confirm and characterize the significance of S321 phosphorylation, we developed a phospho-specific antibody against p-S321 of mouse RIPK1 (anti-p-S321-RIPK1). We probed western blots of cell lysates from mouse embryonic fibroblast (MEF) cells stimulated with TNF $\alpha$ for different periods of time. After stimulation of TNF $\alpha$, the RIPK1 band showed a specific upshift within 5 min TNF $\alpha$ stimulation and the shift reduced after 30-60 min treatment. This shifted band was recognized by anti-pS321-RIPK1 (Fig. 1b). The phosphorylation of IKK $\beta$, known to be mediated by $\mathrm{TAK} 1^{13}$, was also detected during the same time frame. Induction of RIPK1 S321 phosphorylation was also found in RGC- 5 cells stimulated by TNF $\alpha$ (Supplementary Fig. 1a). To confirm that the band recognized by anti-p-S321-RIPK1 is RIPK1, we immunoprecipitated RIPK1 from cells stimulated by TNF $\alpha$ and probed the immunocomplexes with anti-p-S321RIPK1. As shown in Fig. 1c, anti-p-S321-RIPK1 specifically recognized immunoprecipitated RIPK1 from TNF $\alpha$-stimulated cells but not from control cells. In addition to MEF and RGC-5 cells, we verified TNF $\alpha$ induced RIPK1 phosphorylation in primary cells. As that observed in MEFs and RGC-5 cells, in bone marrow-derived macrophages (BMDM) and mouse primary microglia stimulated by $\mathrm{TNF} \alpha$, the phosphorylation of RIPK1 S321 occurred in a similar pattern as that of IKK $\beta$ (Fig. 1d, e). Thus, S321 of RIPK1 is phosphorylated transiently after TNF $\alpha$ stimulation. 
TAK1 phosphorylates RIPK1 S321. On the basis of the temporal profile of RIPK1 S321 phosphorylation upon stimulation by TNF $\alpha$, we focused on characterizing the involvement of TAK1 and TBK1 as possible kinases responsible for phosphorylating RIPK1 S321 in response to TNF $\alpha$ stimulation. We treated L929 and BV-2 cells with TNF $\alpha$ in the presence of TAK1 inhibitor (5Z7-Oxozeaenol, 5Z-7) or TBK1 inhibitor (BX795). The treatment with either TAK1 or TBK1 inhibitors blocked TNF $\alpha$-stimulated S321 phosphorylation in L929 cells (Supplementary Fig. 1b), but only TAK1 inhibitor blocked the phosphorylation of S321 in TNF $\alpha$-stimulated BV-2 cells (Fig. 2a). 5Z-7 also inhibited phosphorylation of RIPK1 S321 in RGC-5, MEF, primary BMDM and microglia cells stimulated by TNF $\alpha$ (Supplementary Fig. 1a, $\mathrm{c}$ and Fig. 1d, e). To determine whether TBK1 might mediate the phosphorylation of RIPK1 S321, we used CRISPR-Cas9 technology to generate TBK1 knockout (KO) BV-2 and MEF cells and then stimulated the cells with TNFo. We found that the phosphorylation of RIPK1 S321 was not blocked, but rather enhanced by TBK1 deficiency in both MEF and BV-2 cells (Supplementary Fig. 1c, d). Therefore, we conclude that TBK1 is not the kinase responsible for TNF $\alpha$-induced RIPK1 S321 phosphorylation.

To provide definitive evidence for the role of TAK1 in mediating phosphorylation S321 of RIPK1, we obtained MEFs from mice homozygous for TAK1 $1^{\text {flox/flox }}$ allele $\left(\mathrm{TAK}^{\mathrm{F} / \mathrm{F}}\right)^{14}$. To generate TAK1-deficient MEFs, we excised the floxed genomic fragment by infecting TAK1 ${ }^{\mathrm{F} / \mathrm{F}}$ MEFs with virus expressing Cre and selected single colonies lacking the expression of TAK1 $\left(\mathrm{TAK} 1^{\mathrm{F} / \mathrm{F}} \mathrm{Cre}\right)$. In TAK1 ${ }^{\mathrm{F} / \mathrm{F}}$ Cre MEFs, RIPK1 S321 phosphorylation in response to TNF $\alpha$ was substantially abolished (Fig. 2b). In contrast, the expression of Cre in WT MEF cells did not affect the expression level of TAK1 or RIPK1 S321 phosphorylation in response to TNF $\alpha$ (Fig. 2b), suggesting the blocked RIPK1 S321 phosphorylation in $\mathrm{TAK1}^{\mathrm{F} / \mathrm{F}}$ Cre MEFs was not a result of virus infection or Cre expression per se. Consistently, the phosphorylation levels of p38 MAPK, a downstream target of TAK1 ${ }^{15}$, were also reduced in $\mathrm{TAK}^{\mathrm{F} / \mathrm{F}}$ Cre MEFs but no in WT Cre MEFs (Fig. 2b).

TAK1 activation in response to TNF $\alpha$ is transient, which peaks at 5-10 min and decreases by TAB2-dependent recruitment of protein phosphatases ${ }^{16}$. Deficiency in TAB2 leads to sustained TAK1 activation and sensitized cells to necroptosis ${ }^{8}, 16$. We therefore characterized RIPK1 S321 phosphorylation in WT and TAB2 KO MEFs. We found that compared to that of WT MEFs, the phosphorylation of RIPK1 S321 was enhanced and sustained longer at later time-points in TAB2 KO MEFs when the signals in WT MEFs were already subsided (Fig. 2c). Consistent with the prolonged activation of TAK1, TNF $\alpha$ stimulation in TAB2 KO MEFs also led to sustained phosphorylation of p38 and IKK $\beta$ than that in WT cells.

Next we examined the ability of TAK1 to directly phosphorylate RIPK1 by in vitro kinase assay. To minimize the effect of auto-phosphorylation, we isolated kinase-dead RIPK1 protein, RIPK1 K45M, as a substrate and incubated it with recombinant TAK1 in the presence of ${ }^{32} \mathrm{P}$-ATP. When RIPK1 K45M was incubated alone, only minimal level of phosphorylation could be detected. When incubated with recombinant TAK1, RIPK1 was phosphorylated and the phosphorylation was blocked in the presence of TAK1 inhibitor (Supplementary Fig. 1e). Kinase-dead IKK $\beta$, a well characterized substrate of TAK1 was used as a positive control. Phosphorylation of kinase-dead RIPK1 protein with additional S321A mutation by TAK1 in radioactive kinase assay was reduced but not eliminated (Supplementary Fig. 1e), suggesting the additional TAK1 phosphorylation sites on RIPK1. To further confirm that the TAK1-mediated phosphorylation occurred on S321, we purified RIPK1 WT and S321A mutant proteins and examined whether they could be phosphorylated by
TAK1 in in vitro kinase assay. After incubation with recombinant TAK1, only RIPK1 WT, but not RIPK1 S321A mutant, could be recognized by anti-p-S321-RIPK1 antibody and the phosphorylation was inhibited in the presence of 5Z-7 (Fig. 2d). Thus, TAK1 can directly phosphorylate RIPK1 S321 in vitro.

Recently it was reported that $I K K \alpha / \beta$ could phosphorylate RIPK $1^{5}$. Inhibition of TAK1 by $5 Z-7$ also blocked the activation of IKK $\beta$ occurring downstream of TAK1 in response to TNF $\alpha$ stimulation as expected (Supplementary Fig. 2a). To test the possible contribution of IKK $\alpha / \beta$ to RIPK1 S321 phosphorylation, we examined the effect of IKK $\alpha / \beta$ inhibitor, BMS345541, on RIPK1 S321 phosphorylation. The presence of BMS345541 suppressed IKB $\alpha$ phosphorylation, which is known to be mediated by IKK $\alpha / \beta$ kinase (Supplementary Fig. $2 b)^{17}$. On the other hand, the levels of phospho-RIPK1 S321 and phospho-IKK $\beta$ were sustained even longer in presence of BMS345541 and TNF $\alpha$ (Supplementary Fig. 2b). In addition, we confirmed that recombinant IKK $\alpha$ could phosphorylate RIPK1 in in vitro kinase assay with ${ }^{32}$ P-ATP (Supplementary Fig. 2c) but the phosphorylated RIPK1 could not be recognized by anti-p-S321-RIPK1 antibody (Fig. 2d). Taken together, these results suggest that IKK complex is not involved in mediating the phosphorylation of RIPK1 S321.

The time-course study showed that phosphorylation of RIPK1 S321 occurred within 5 min of TNF $\alpha$ treatment, similar to that of TAK1-mediated IKK $\beta$ phosphorylation (Fig. 1b, d, e). Since TAK1 is transiently recruited to TNF-RSC within 5 min after TNF $\alpha$ treatment, we next tested if the phosphorylation of RIPK1 S321 occurred in TNF-RSC. MEF cells were treated with FLAGtagged TNF $\alpha$ and TNF-RSC was purified by anti-FLAG immunoprecipitation. Consistent with the phosphorylation by TAK1, we detected the S321 phosphorylation of both high molecular weight ubiquitinated and un-ubiquitinated RIPK1 species in TNF-RSC, which was inhibited by 5Z-7 (Fig. 2e). Taken together, we conclude that TAK1 mediates the phosphorylation of RIPK1 S321 in TNF-RSC upon TNF $\alpha$ stimulation.

S321 phosphorylation of RIPK1 requires cIAP1/2. CIAP1mediated K63 ubiquitination of RIPK1 is known to be critical for the recruitment of TAK1 into the TNF-RSC upon TNF $\alpha$ stimulation $^{18}$. To examine the role of K63 ubiquitination on RIPK1 in TNF $\alpha$-stimulated S321 phosphorylation, we treated cells with SM-164, a small molecule IAP antagonist that can promote the degradation of $c I A P 1 / 2^{19}$. TNF $\alpha$-induced RIPK1 ubiquitination in TNF-RSC was substantially reduced in the presence of SM-164 (Supplementary Fig. 3a). Accordingly, TNF $\alpha$-induced RIPK1 S321 phosphorylation was abolished after SM-164 treatment (Fig. 3a). Furthermore, S321 phosphorylation was not detected in TNF $\alpha$-stimulated cIAP1/2 double knockout (DKO) cells (Fig. 3b). Thus, cIAP1/2 is required for phosphorylation of RIPK1 S321 induced by TNF $\alpha$.

To further characterize the requirement for RIPK1 ubiquitination in TAK1-mediated phosphorylation of S321, we examined the requirement of TRADD, which is involved in the recruitment of cIAP1/2 and TRAF2 to TNFR1, and TRAF2, an adaptor protein for the recruitment of cIAP1/20. As shown in Fig. 3b, the phosphorylation of S321 stimulated by TNF $\alpha$ was not detectable in TRADD KO, or TRAF2 KO MEF cells. Consistent with the requirement of K63 ubiquitination in phosphorylation of S321, treatment with TAK1 inhibitor in the presence of SM-164 did not further sensitize cells to TNF $\alpha$-induced cell death (Supplementary Fig. 3b). Taken together, these results suggest that cIAP1mediated K63 ubiquitination of RIPK1 is important for promoting phosphorylation of RIPK1 S321. 
a

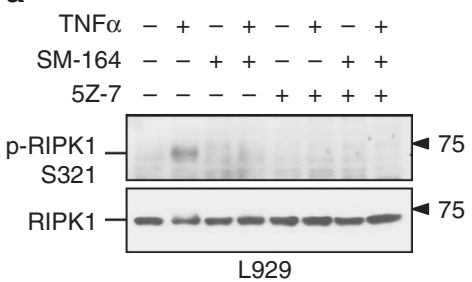

C

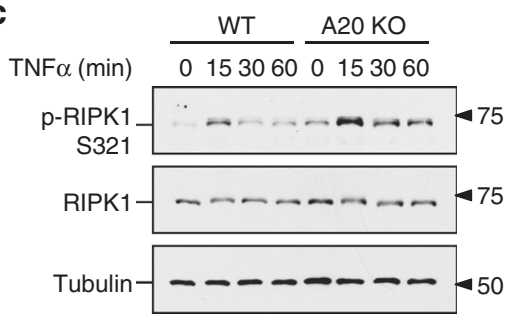

b

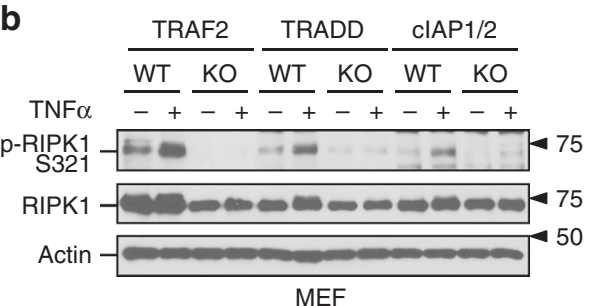

MEF

Fig. 3 Ubiquitination of RIPK1 is essential for its phosphorylation by TAK1. a SM-164 treatment blocks RIPK1 S321 phosphorylation in L929 cells. The cells were pre-treated by SM-164 (50 nM) and/or 5Z-7 (0.5 $\mu$ M) as indicated for $30 \mathrm{~min}$, and incubated with TNF $\alpha$ (10 ng/ml) for $5 \mathrm{~min}$. b RIPK1 S321 is not phosphorylated in the absence of TRAF2, TRADD or CIAP1/2. TRAF2, TRADD, cIAP1/2 KO MEFs and their corresponding WT MEFs were treated with TNF $\alpha$ for 5 min. c RIPK1 S321 hyperphosphorylation in A2O KO MEFs. A2O KO and WT MEFs were treated with TNF $\alpha$ and samples were collected at indicated time-points. The phosphorylation of RIPK1 S321 was analyzed by western blot using indicated antibodies a-c

Since A20 is a critical ubiquitin-editing enzyme that can move K63 ubiquitin chain from RIPK1 in TNF $\alpha$-stimulated cells to down-regulate TNF $\alpha$ signaling ${ }^{21}$, we examined the role of A20 in regulating S321 phosphorylation of RIPK1. We found that TNF $\alpha$ induced RIPK1 S321 phosphorylation in A20-deficient cells was significantly higher and persisted longer than that in WT cells (Fig. 3c). Given the fact that A20-deficient cells are hypersensitive to RIPK1 activation and necroptosis 22 , this result suggests that the possibility of elevated phosphorylation on RIPK1 by TAK1 may regulate the activation of RIPK1.

Phosphorylation of RIPK1 S321 regulates RDA. Next, we explored the biological significance of RIPK1 S321 phosphorylation. To determine whether RIPK1 S321 phosphorylation occurs in vivo, we stimulated mice with $\mathrm{TNF} \alpha$ via intraperitoneal injections and characterized the phosphorylation of RIPK1 by western blot. We found that TNF $\alpha$ stimulation in vivo was able to induce the phosphorylation of RIPK1 S321 in the liver, kidney, intestine and spleen (Fig. 4a). To determine the significance of RIPK1 S321 phosphorylation in vivo, we compared the effects of virally transduced RIPK1 WT, S321A (SA) and S321E (SE) expression in the liver, delivered using adeno-associated virus (AAV) vector, a small single-stranded DNA-containing nonpathogenic human parvovirus that has been used as an efficient vehicle for gene transfer to different tissues including liver without apparent vector-related toxicities ${ }^{23}$. Specifically, we constructed AAV vectors expressing FLAG-tagged RIPK1 WT, S321A and S321E under the control of a liver-specific promoter $\mathrm{TBG}^{24}$. We followed the effect of RIPK1 expression in C57BL/6 mice intravenously injected with RIPK1 expression AAVs using plasma levels of alanine aminotransferase (ALT), a wellestablished biomarker for liver damage. C57BL/6 mice are known to be tolerogenic to rAAV gene delivery to the liver ${ }^{23}$. Only a basal level of ALT release was found in the plasma of mice received control (GFP-expressing) AAV. Interestingly, while low levels of ALT release were detected in the plasma of mice transduced with RIPK1 WT, significantly elevated levels of ALT were found in the plasma of mice that received RIPK1 S321A, but not S321E, AAV (Fig. 4b). Furthermore, the levels of TNF $\alpha$ were also significantly higher in the liver tissues of mice that received
RIPK1 S321A virus (Fig. 4c). Importantly, the increased release of ALT and TNF $\alpha$ was inhibited in mice treated with Nec-1s (Fig. 4b, c). The effect of RIPK1 S321A virus to induce liver damage was directly verified using TUNEL staining (Fig. 4d). The expression level of FLAG-RIPK1 S321A was comparable to those of WT and S321E, suggesting their different physiological effect was not due to variations in expression levels (Fig. 4e). The cleavage of RIPK1 S321A was elevated than that of WT in vivo, while the cleavage of RIPK1 S321E was reduced (Fig. 4e). Thus, the expression of S321A was more effective than that of RIPK1 WT or S321E in inducing RIPK1-dependent liver damage and inflammation in vivo.

To better understand the significance of RIPK1 S321 phosphorylation in regulating cell death, we generated knock-in MEFs carrying RIPK1 S321A or S321E mutations by CRISPRCas9 technology. Phosphorylation of S321 after TNFo stimulation was eliminated in S321A(A/A) MEFs (Supplementary Fig. 4a). S321A(A/A) or S321E(E/E) MEFs showed no difference in the phosphorylation of $I K K \alpha / \beta$, or in the phosphorylation or degradation of $\mathrm{I} \kappa \mathrm{B} \alpha$ in response to $\mathrm{TNF} \alpha$ stimulation (Supplementary Fig. 4b), suggesting that S321 mutation has no effect on TNF $\alpha$-stimulated NF- $\kappa$ B activation. Recruitment of CYLD, HOIP and Sharpin to TNF-RSC in response to TNF $\alpha$ was not affected in RIPK1 S321A(A/A) MEFs (Supplementary Fig. 4c). TNF $\alpha-$ induced RIPK1 recruitment and ubiquitination in TNF-RSC was slightly increased in RIPK1 S321A(A/A) MEFs (Supplementary Fig. 4d). On the other hand, homozygous S321A(A/A) MEFs showed increased sensitivity to cell death induced by $\mathrm{TNF} \alpha$, $\mathrm{TNF} \alpha /$ cycloheximide $(\mathrm{CHX})$ or $\mathrm{TNF} \alpha / \mathrm{CHX} / \mathrm{zVAD}$.fmk (zVAD) and Nec-1s protected S321A(A/A) MEFs to all three treatments (Fig. 5a-c). For better quantification of apoptosis, WT and S321A (A/A) MEFs were stained with SYTOX Green after TNF $\alpha$ alone or TNF $\alpha / C H X$ treatment. As shown in Fig. 5d and Supplementary Fig. 5a, S321A(A/A) MEFs were more sensitive than WT cells to apoptosis induced by $\mathrm{TNF} \alpha$ or $\mathrm{TNF} \alpha / \mathrm{CHX}$ and $\mathrm{Nec}-1$ s only protected cell death in S321A(A/A) MEF cells. While TNF $\alpha / C H X$ normally promotes RIPK1-independent apoptosis in WT cells, cell death of RIPK1 S321A(A/A) MEFs induced by TNF $\alpha$ alone or TNF $\alpha / \mathrm{CHX}$ was inhibited by Nec-1s, suggesting that the lack of S321 phosphorylation sensitizes cells to TNF $\alpha$-induced RDA. 
a

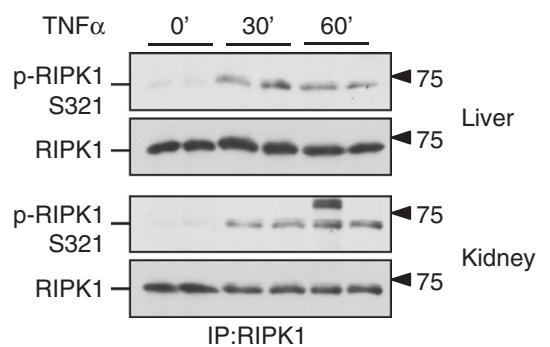

b

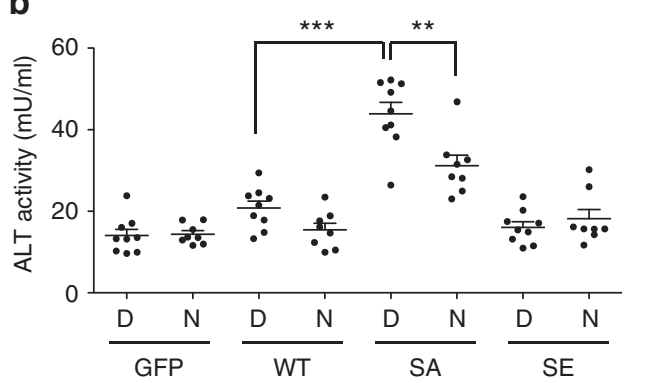

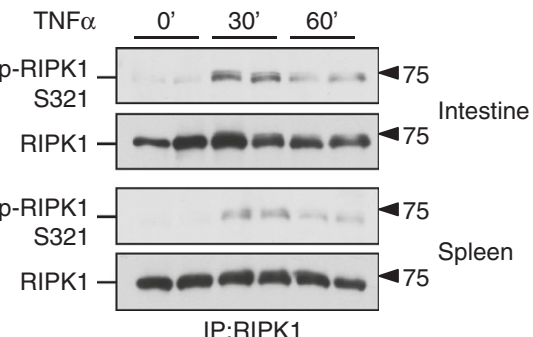

C

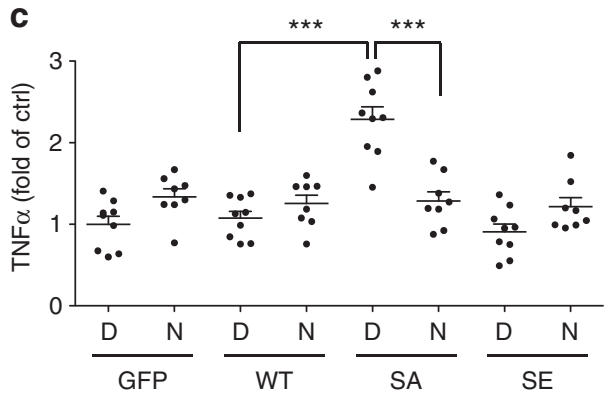

d

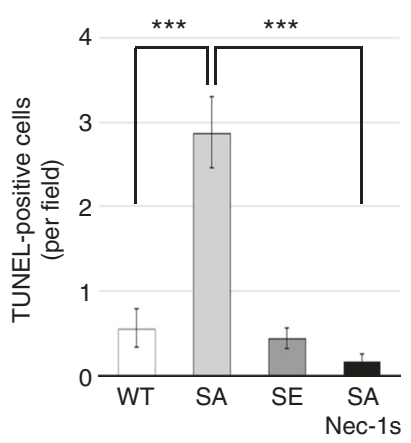

e

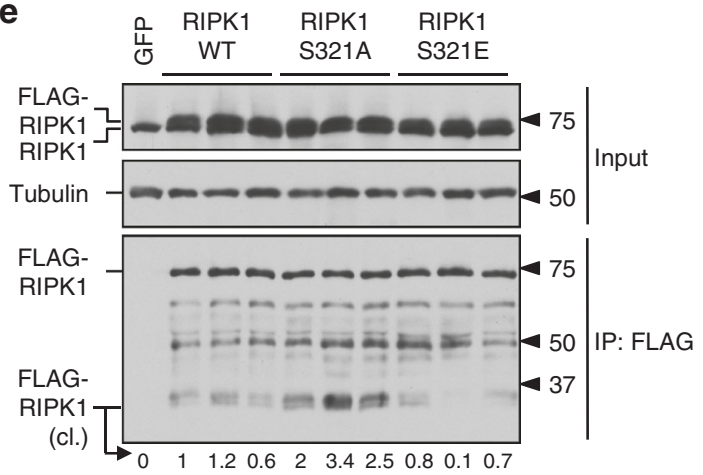

Fig. 4 AAV-mediated RIPK1 S321A mutant expression induces liver damage in vivo. a Phosphorylation of RIPK1 S321 in mice tissues after TNF $\alpha$ injection. $30 \mu \mathrm{g}$ TNF $\alpha$ were delivered to mice (8-week-old male) through intraperitoneal injections. Tissues were collected at 30 and 60 min after the injection. RIPK1 was immunoprecipitated from tissue lysates by RIPK1 total antibody. b, $\mathbf{c}$ Liver damage in mice induced by AAV-mediated RIPK1 S321A mutant expression. AAVs carrying RIPK1 WT, S321A, S321E or GFP as a control were delivered through tail vein injection. Sixteen days after the AAV injection, the mice were treated with DMSO $(D, n=9)$ or Nec-1s $(N, n=8)$ through oral dosing. Four weeks after the AAV injection, plasma and liver samples were collected. ALT $\mathbf{b}$ and TNF $\alpha$ ELISA $\mathbf{c}$ assays were performed as manufacture's instructions. d TUNEL staining for assessing liver damage induced by AAV-mediated RIPK1 expression. Liver sections were stained with TUNEL and Hoechst. Cells with co-localized TUNEL and Hoechst signals were counted as TUNEL-positive. e AAV-mediated expression of FLAG-RIPK1 variants in liver. The liver lysates were subjected to anti-FLAG immunoprecipitation to differentiate endogenous RIPK1 and ectopic FLAG-RIPK1. Relative intensity of cleaved (cl.) RIPK1 bands was quantified. Error bar, s.e.m. ${ }^{\star \star}, t$-test $P<0.01,{ }^{\star \star \star} P<0.001$. Three independent repeats were included in each data point

To verify the induction of RDA, we investigated the activation of caspases and RIPK1 in S321A(A/A) MEFs. We found that the activity of caspase-8 in S321A(A/A) MEFs, but not WT or S321E (E/E) MEFs, was stimulated by TNF $\alpha$ alone (Fig. 5e). Consistent with the activation of RDA, the addition of Nec-1s inhibited the activation of caspase-8. Following the evidence of enhanced caspase- 8 activity, we examined the cleavage of RIPK1 and CYLD, two well-established substrates of caspase-8. Augmented cleavage of RIPK1 and CYLD was detected in S321A(A/A) compared to WT MEFs after TNF $/$ CHX treatment (Supplementary Fig. 5b). While the cleavage of CYLD and RIPK1 in both WT and S321A(A/A) MEFs was inhibited by pan-caspase inhibitor $\mathrm{zVAD}$, the addition of $\mathrm{Nec}-1 \mathrm{~s}$ was only able to inhibit the cleavage of CYLD and RIPK1 in S321A(A/A) MEFs, but not in WT MEFs, which further supports the induction of RDA in S321A(A/A) MEFs.

RIPK1 is known to be cleaved after D324 in human RIPK1 (its equivalent in murine RIPK1 is D325) by caspase- 8 during TNF $\alpha$ mediated apoptosis ${ }^{25}$. Since S321 is only one amino acid beyond the usual 4-amino acid motif required for caspase recognition, we tested if the phosphorylation of S321 or its single point mutant might affect the cleavage by caspase- 8 in vitro. However, S321A mutation had no effect on the cleavage by caspase- 8 in vitro while S321E only showed weak increase of cleavage in vitro (Supplementary Fig. 5c). Thus, instead of determining the sensitivity of RIPK1 as a substrate for caspase-8, the phosphorylation of S321 is involved in the regulation of caspase- 8 activation.

RIPK1 S321A mutation promotes its binding with FADD. Phosphorylation of RIPK1 S166 has been established as a biomarker of RIPK1 activation ${ }^{11,26,27}$. To determine if the activation of RIPK1 might be affected by the phosphorylation of S321, we compared the phosphorylation of RIPK1 S166 in WT and $\mathrm{S} 321 \mathrm{~A}(\mathrm{~A} / \mathrm{A}) \mathrm{MEF}$ stimulated by $\mathrm{TNF} \alpha, \mathrm{TNF} \alpha / \mathrm{CHX}$ or $\mathrm{TNF} \alpha /$ $\mathrm{CHX} / \mathrm{zVAD}$. Stimulation of $\mathrm{TNF} \alpha / \mathrm{CHX} / \mathrm{zVAD}$ is known to activate RIPK1 and necroptosis ${ }^{26}$. Consistently, the phosphorylation of RIPK1 S166 in WT MEFs was only detected upon 
a
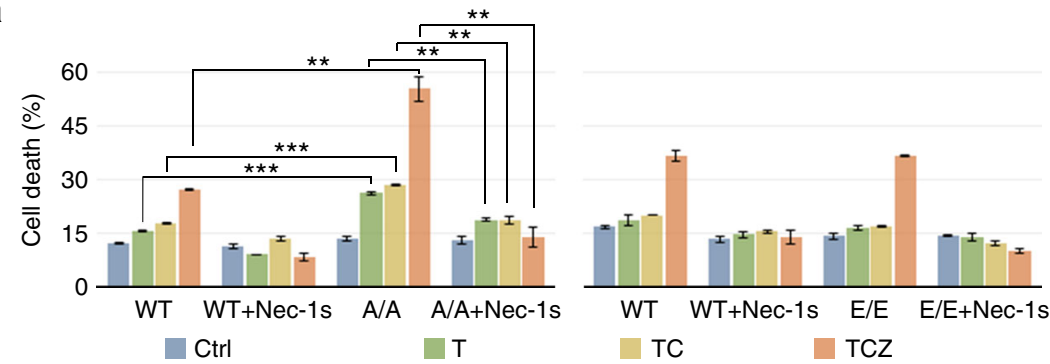

b

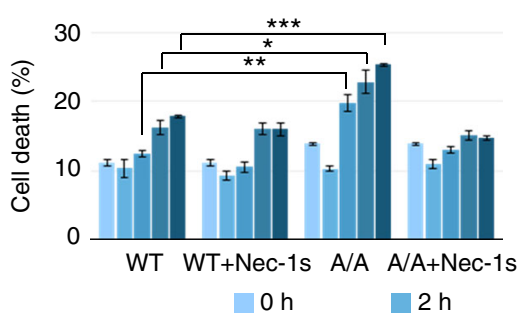

C

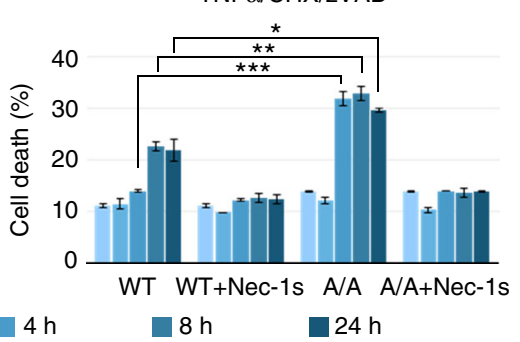

d

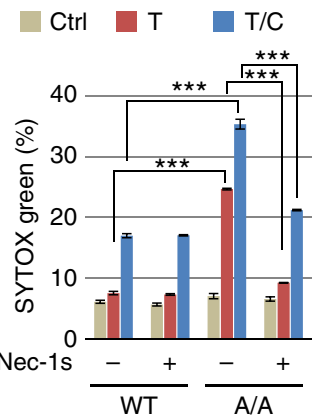

e $\quad \mathrm{Ctrl}$

TNF $\alpha+N e c-1 s$

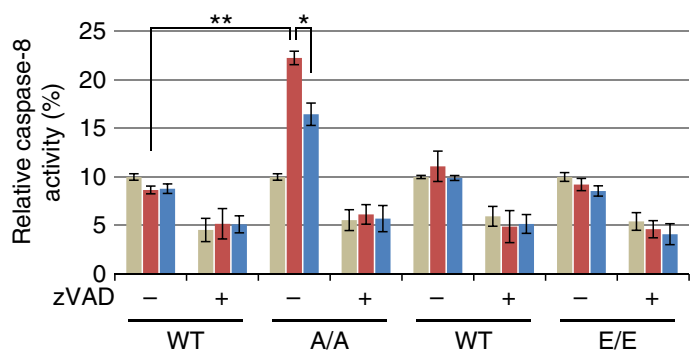

Fig. 5 RIPK1 S321A mutation sensitizes cells to RIPK1-dependent cell death. a-c RIPK1 S321A(A/A) but not S321E(E/E) MEFs are more sensitive to RIPK1dependent cell death. Immortalized MEFs generated from littermates of WT and RIPK1 S321A(A/A), or WT and S321E (E/E) were treated with TNF $\alpha$ $(10 \mathrm{ng} / \mathrm{ml}), \mathrm{CHX}(0.5 \mu \mathrm{g} / \mathrm{ml}), \mathrm{zVAD}(20 \mu \mathrm{M})$ and Nec-1s $(10 \mu \mathrm{M})$ as indicated. Cell death was measured by ToxiLight assay after $16 \mathrm{~h}$ treatment a or at indicated time-points b, $\mathbf{c}$ and normalized to TX-100-treated cells. d TNF $\alpha$ alone or TNF $\alpha / C H X$ induces RDA in RIPK1 S321A(A/A) mutant MEFs. WT and RIPK1 S321A(A/A) MEFs were treated with TNF $\alpha(10 \mathrm{ng} / \mathrm{ml})$ or CHX $(0.5 \mu \mathrm{g} / \mathrm{ml})$ with or without Nec-1s $(10 \mu M)$ for $24 \mathrm{~h}$. After SYTOX Green staining, fluorescence intensity was quantified and normalized to TX-100-treated cells. e TNF $\alpha$ induces caspase-8 activation in RIPK1 S321A(A/A) mutant but not in WT and S321E(E/E) mutant MEFs. The MEFs were treated with TNF $\alpha(10 \mathrm{ng} / \mathrm{ml})$ with or without Nec-1s $(20 \mu \mathrm{M})$ for $24 \mathrm{~h}$ and caspase-8 activity was measured in the presence or absence of ZVAD as described in Methods. Error bar, s.e.m. ${ }^{\star}, t$-test $P<0.05 ;{ }^{\star \star} P<0.01,{ }^{\star \star \star} P<0.001$. Three independent repeats were included in each data point

stimulation by $\mathrm{TNF} \alpha / \mathrm{CHX} / \mathrm{zVAD}$ together, but not by $\mathrm{TNF} \alpha /$ CHX or TNF $\alpha$ alone (Fig. 6a). On the other hand, the phosphorylation of RIPK1 S166 in S321A(A/A) MEFs stimulated by $\mathrm{TNF} \alpha / \mathrm{CHX} / \mathrm{zVAD}$ was significantly higher than that of WT, and furthermore, it was also detectable when stimulated by TNF $\alpha /$ CHX or TNFo alone (Fig. 6a). In addition, K63 ubiquitination of RIPK1 in TNF $\alpha$-stimulated S321A(A/A) MEFs also increased (Fig. 6b). These results suggest that TNF $\alpha$ or TNF $\alpha / C H X$ treatment can promote the activation of RIPK1 when S321 phosphorylation is blocked. Given the increased caspase activity and RIPK1 S166 phosphorylation in S321A(A/A) MEFs, we concluded that blocking the phosphorylation of RIPK1 S321 sensitized cells to RDA upon stimulation by TNF $\alpha$ or $\mathrm{TNF} \alpha / \mathrm{CHX}$, which was not sufficient to induce the activation of RIPK1 in WT cells.

Consistent with increased activation of RIPK1, S321A(A/A) MEFs also showed an increased sensitivity to necroptosis induced by $\mathrm{TNF} \alpha / \mathrm{CHX} / \mathrm{zVAD}$, which was inhibited by the addition of Nec-1s (Fig. 5a, c). On the other hand, the phosphorylation of MLKL and the interaction of RIPK1 and RIPK3 to form complex $\mathrm{IIb}$, the hallmarks of necroptosis, were only detected in S321A(A/
A) MEFs when stimulated by $\mathrm{TNF} \alpha / \mathrm{CHX} / \mathrm{zVAD}$, but not by TNF $\alpha / C H X$ (Fig. 6c, d). Thus, S321A mutation alone is not sufficient to activate necroptosis without caspase inhibition.

Since the activation of caspase- 8 is mediated by the interaction of RIPK1 with FADD, we next characterized the interaction of RIPK1 and FADD in WT and S321A(A/A) MEFs by coimmunoprecipitation. We found that compared to that of WT MEFs, the interaction of RIPK1 and FADD in S321A(A/A) MEFs after treatment with $\mathrm{TNF} \alpha / \mathrm{CHX}$ was significantly increased. Furthermore, the treatment with Nec-1s inhibited the interaction of FADD and RIPK1 in S321A(A/A) MEFs but not in WT MEFs induced by TNF $\alpha / \mathrm{CHX}$ (Fig. 6e). Taken together, we conclude that TAK1-mediated S321 phosphorylation on RIPK1 negatively regulates its activation and interaction with FADD. Thus, blocking S321 phosphorylation of RIPK1 sensitizes cells to TNF $\alpha$-induced RDA by promoting the interaction of RIPK1 and FADD in a manner regulated by the kinase activity of RIPK1.

RIPK1 hyperphosphorylation promotes necroptosis. Hyperactivation of TAK1 in TAB2 KO MEF has been shown to sensitize cells to necroptosis ${ }^{8}$; however, the mechanism is unclear. 
a

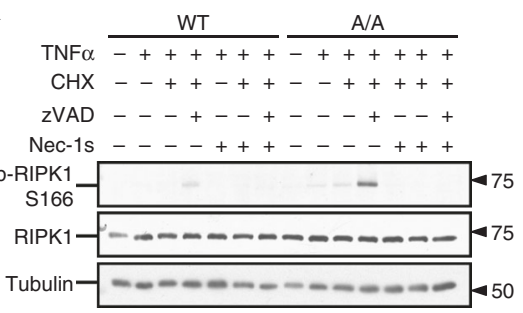

C

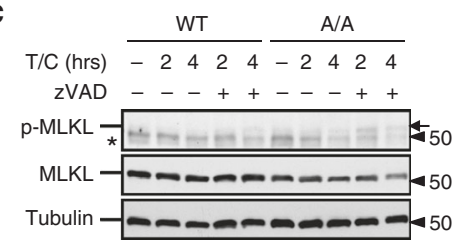

d

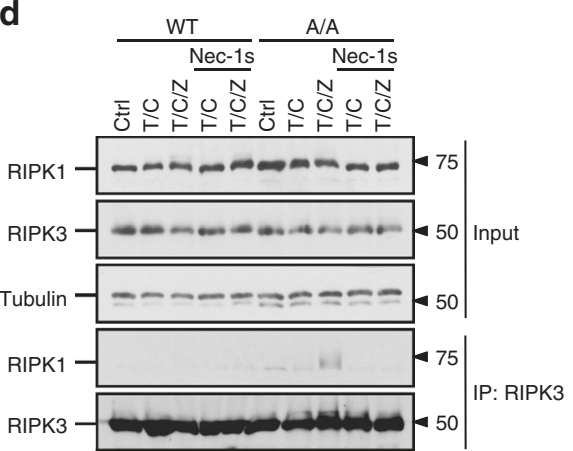

b
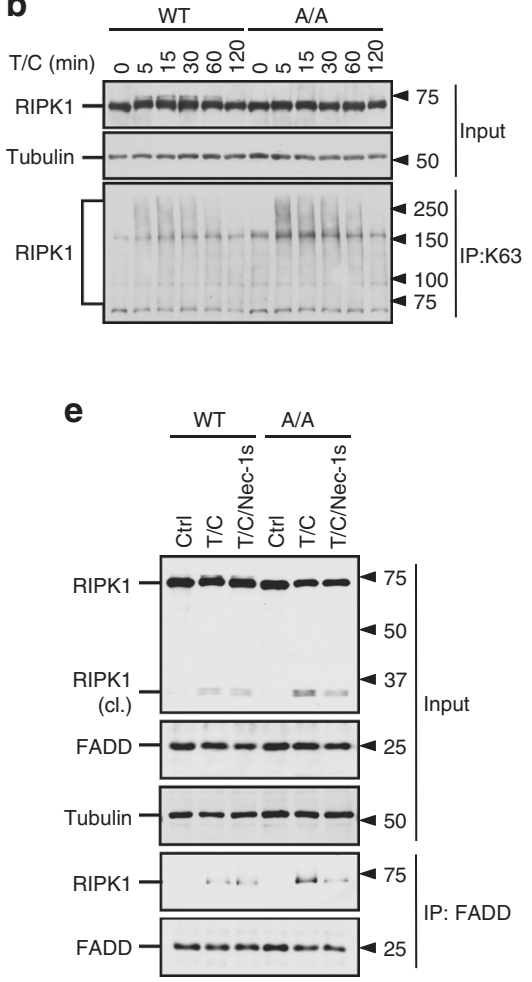

Fig. 6 S321A mutation promotes RIPK1 activation. a RIPK1 S166 phosphorylation in S321A(A/A) mutant. WT and S321A(A/A) MEFs were treated with TNF $\alpha(50 \mathrm{ng} / \mathrm{ml}), \mathrm{CHX}(1 \mu \mathrm{g} / \mathrm{ml}), \mathrm{zVAD}(20 \mu \mathrm{M})$ and Nec-1s $(10 \mu \mathrm{M})$ as indicated for $2 \mathrm{~h}$. b S321A mutation augments RIPK1 K63 ubiquitination in response to TNF $\alpha / \mathrm{CHX}$ treatment. MEFs were treated with TNF $\alpha(50 \mathrm{ng} / \mathrm{ml})$ and $\mathrm{CHX}(1 \mu \mathrm{g} / \mathrm{ml})$. Lysates under denaturing condition were collected at indicated time-points, immunoprecipitated with $\mathrm{K} 63$ antibody and detected with RIPK1 antibody. c Enhanced MLKL phosphorylation in S321A(A/A) cells in response to TNF $\alpha / C H X / z V A D$. MEFs were treated with TNF $\alpha(10 \mathrm{ng} / \mathrm{ml}), \mathrm{CHX}(1 \mu \mathrm{g} / \mathrm{ml}), \mathrm{zVAD}(20 \mu \mathrm{M})$ for 2 and $4 \mathrm{~h}$. Arrow, phosphorylated $\mathrm{MLKL}$. d Earlier induction of RIPK1-RIPK3 interaction in S321A(A/A) MEFs induced by TNF $\alpha / C H X / z V A D$. WT and RIPK1 S321A(A/A) MEFs were treated as a and co-immunoprecipitation was performed with RIPK3 antibody. e Stronger interaction between FADD and RIPK1 in RIPK1 S321A(A/A) MEFs induced by TNF $\alpha / \mathrm{CHX}$ was suppressed by Nec-1s. The cells were treated with TNF $\alpha(50 \mathrm{ng} / \mathrm{ml})$ and $\mathrm{CHX}(1 \mu \mathrm{g} / \mathrm{ml})$ with or without Nec-1s (20 $\mu \mathrm{M})$ for $4 \mathrm{~h}$. Coimmunoprecipitation was performed with FADD antibody. ${ }^{*}$, nonspecific band

Given the enhanced phosphorylation on RIPK1 S321 in TAB2 KO cells, we hypothesized that hyperphosphorylation of RIPK1 by TAK1 might promote necroptosis. To test this hypothesis, we first characterized complex IIa formation and RIPK1 cleavage in S321E(E/E) MEFs induced by TNF $\alpha / \mathrm{CHX}$. When treated with $\mathrm{TNF} \alpha / \mathrm{CHX}$, caspase-8-dependent RIPK1 cleavage was significantly reduced in $\mathrm{S} 321 \mathrm{E}(\mathrm{E} / \mathrm{E}) \mathrm{MEF}$ cells compared to WT (Fig. 7a). Furthermore, RIPK1 was co-immunoprecipitated with FADD in WT cells but not in S321E(E/E) mutant after $4 \mathrm{~h} \mathrm{TNF} \alpha /$ CHX treatment (Fig. 7a). Although the pro-apoptosis complex IIa was suppressed, RIPK1 S321E(E/E) MEFs were not more sensitive to $\mathrm{TNF} \alpha / \mathrm{CHX} / \mathrm{zVAD}$-induced necroptosis compared to WT cells (Fig. 5a). Thus, increased phosphorylation of RIPK1 S321 alone might not be sufficient to drive necroptosis.

RIPK1 can be phosphorylated in a number of Ser residues close to S321, e.g., S332 and S334 when expressed in $293 \mathrm{~T}_{\text {cells }}{ }^{11}$. Since the phosphorylation of S332/334 was not affected by RIPK1 kinase-dead K45M mutation, they were unlikely to be sites of auto-phosphorylation. With additional S332/334/336A mutation, RIPK1 phosphorylation by recombinant TAK1 in in vitro kinase assay was further attenuated compared to kinase-dead RIPK1 S321A mutant (Supplementary Fig. 1e). To investigate the kinase mediating RIPK1 S332/334 phosphorylation, we generated an antibody against phosphorylation on these sites. Using this antibody, we found that the phosphorylation of S332/334 RIPK1 was also detectable in early time-points after TNF $\alpha$ stimulation and the signal was inhibited by $5 Z-7$, suggesting that they might also be phosphorylated by TAK1 (Fig. 7b). Furthermore, the signal detected by this p-S332/334 RIPK1 antibody was eliminated by S321/332/334/336A (AAAA) mutation (Fig. 7c).

To characterize the effect of additional TAK1 phosphorylation sites on RIPK1, we mutated these three TAK1 sites together with S321 to generate RIPK1 S321/332/334/336E (EEEE) quadruple mutant as a model for sustained RIPK1 phosphorylation in the intermediate domain. We found that the expression of RIPK1 EEEE mutant in RGC-5 RIPK1 KO cells was sufficient to induce spontaneous RIPK1 kinase activation as shown by the detection of RIPK1 S166 phosphorylation and its interaction with RIPK3 in the absence of TNFo stimulation (Fig. 7d). Increased phosphorylation of MLKL was detected in RGC-5 cells expressing RIPK1 EEEE mutant (Fig. 7e). As a result, RIPK1 EEEE mutant induces spontaneous cell death which could be protected by RIPK3 knockdown or treatment of RIPK3 inhibitor (Fig. 7f). The expression level of RIPK1 EEEE mutant was comparable to that of other RIPK1 variants (Fig. 7d, e and Supplementary Fig. 6b), so the activation of RIPK1 and RIPK3 as well as consequent cells death were not due to higher expression of RIPK1 EEEE mutant. Taken together, these results suggest that sustained RIPK1 phosphorylation in the intermediate domain promotes its interaction with RIPK3 to drive necroptosis (Fig. 8). 
a

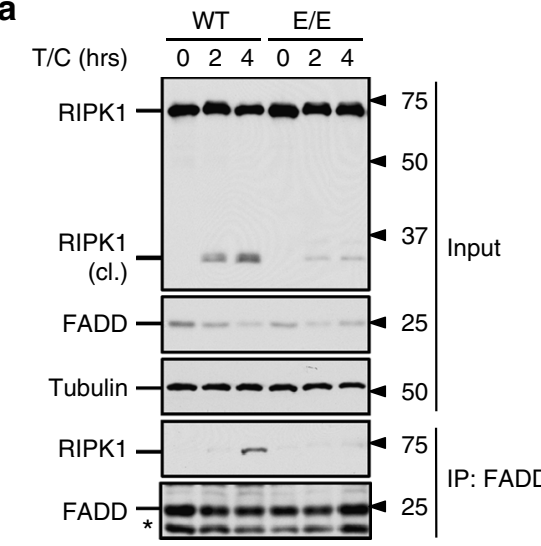

C

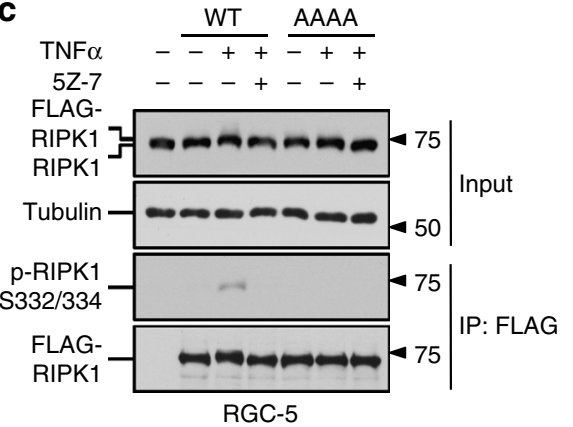

b

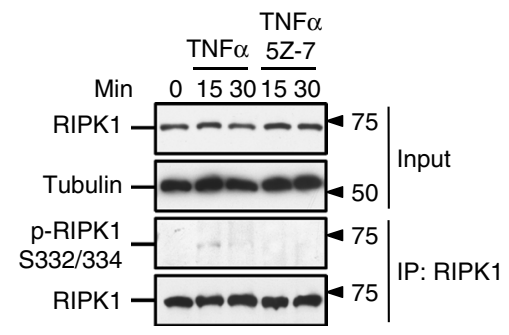

d

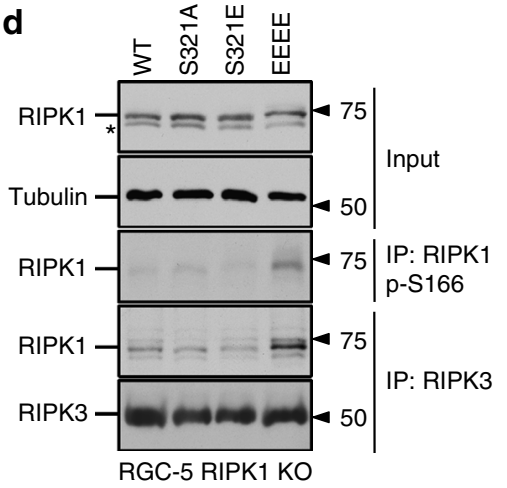

e

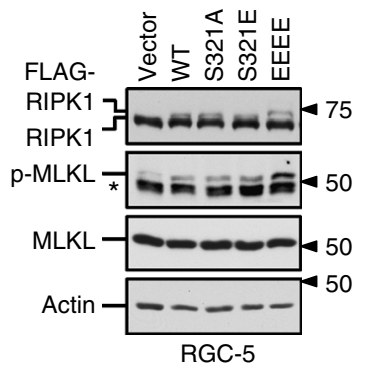

f

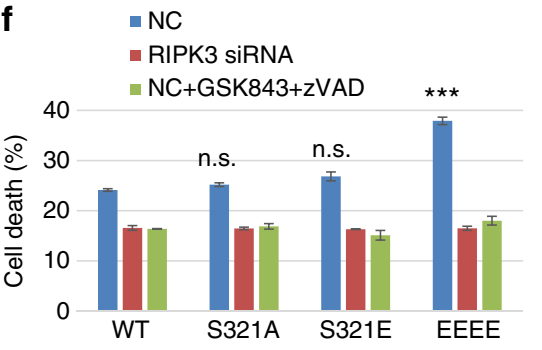

Fig. 7 Hyperactivation of RIPK1 mediated by TAK1 sensitizes cells to necroptosis. a S321E mutation suppresses RIPK1 cleavage and RIPK1-FADD interaction induced by $\mathrm{TNF} \alpha / \mathrm{CHX}$. The cells were treated with $\mathrm{TNF} \alpha(50 \mathrm{ng} / \mathrm{ml})$ and $\mathrm{CHX}(1 \mu \mathrm{g} / \mathrm{ml})$ for 2 or $4 \mathrm{~h}$. Co-immunoprecipitation was performed with FADD antibody. b TNF $\alpha$ induces RIPK1 S332/334 phosphorylation. RGC-5 cells were treated TNF $\alpha(10 \mathrm{ng} / \mathrm{ml})$ with or without $5 Z-7$ (0.5 $\mu$ M). RIPK1 was isolated by immunoprecipitation and detected by p-RIPK1 S332/334 and RIPK1 total antibodies. c RIPK1 S321/332/334/336A (AAAA) mutation blocks TNF $\alpha$ induced S332/334 phosphorylation. FLAG-RIPK1 WT, AAAA mutant or empty vector was transiently expressed in RGC-5 cells and treated with TNF $\alpha$ $(10 \mathrm{ng} / \mathrm{ml})$ and $5 Z-7(0.5 \mu \mathrm{M})$ for $15 \mathrm{~min}$. FLAG-RIPK1 was purified by anti-FLAG immunoprecipitation and detected by p-RIPK1 S332/334 and RIPK1 total antibodies. d RIPK1 kinase activity and its interaction with RIPK3 were induced by RIPK1 S321/332/334/336E (EEEE) mutant overexpression. RGC-5 RIPK1 KO cells were transfected with RIPK1 WT and mutants. $30 \mathrm{~h}$ after transfection, the cells were lysed and immunoprecipitated with RIPK1 p-S166 or RIPK3 antibodies and blotted with RIPK1 total antibody. e Transient expression of RIPK1 EEEE mutant promotes MLKL phosphorylation in RGC-5 cells. RGC-5 cells were transfected with FLAG-RIPK1 variants and samples were collected $24 \mathrm{~h}$ after transfection. $\mathbf{f}$ Spontaneous necroptosis in response to the expression of RIPK1 EEEE mutant. RGC-5 RIPK1 KO cells with or without RIPK3 knockdown were transfected with RIPK1 WT or mutants. GSK843 (10 $\mu$ M)/zVAD (20 $\mu \mathrm{M})$ was added as indicated. Cell death was measured by ToxiLight assay $24 \mathrm{~h}$ after transfection. ${ }^{\star}$, nonspecific bands. Error bar, s.e.m. ${ }^{\star \star \star}, t$-test $P<0.001$; n.s., not significant. Three independent repeats were included in each data point

\section{Discussion}

In this manuscript, we demonstrate a novel mechanism by which phosphorylation of the intermediate domain of RIPK1 by TAK1 dictates alternative cell death mechanisms. We show that dysregulation of RIPK1 phosphorylation by TAK1, including both inhibition or hyperphosphorylation, promotes the activation of RIPK1. On the other hand, TAK1-mediated phosphorylation in the intermediate domain of RIPK1 dictates whether RIPK1 interacts with FADD to form the complex IIa to mediate apoptosis in RIPK1-dependent or -independent manner, or with RIPK3 to form the necrosome (complex IIb) to drive necroptosis. Specifically, the lack of RIPK1 S321 phosphorylation by TAK1 promotes its interaction with FADD to mediate RDA; whereas excessive phosphorylation of RIPK1 by TAK1 in the intermediate domain promotes its interaction with RIPK 3 while suppressing its binding with FADD to promote necroptosis. In WT cells, TNF $\alpha$ induced transient phosphorylation of RIPK1 by TAK1 in the presence of CHX is sufficient to block the activation of RIPK1 and drives RIPK1-independent apoptosis. On the other hand, the absence of S321 phosphorylation on RIPK1 in TNF $\alpha$ alone or $\mathrm{TNF} \alpha / \mathrm{CHX}$-stimulated S321A(A/A) cells promotes the activation of RIPK1 and RIPK1-dependent caspase activation to mediate RDA. Finally, the sustained phosphorylation of RIPK1 by TAK1 modeled by RIPK1 EEEE mutant suppresses RIPK1-FADD 


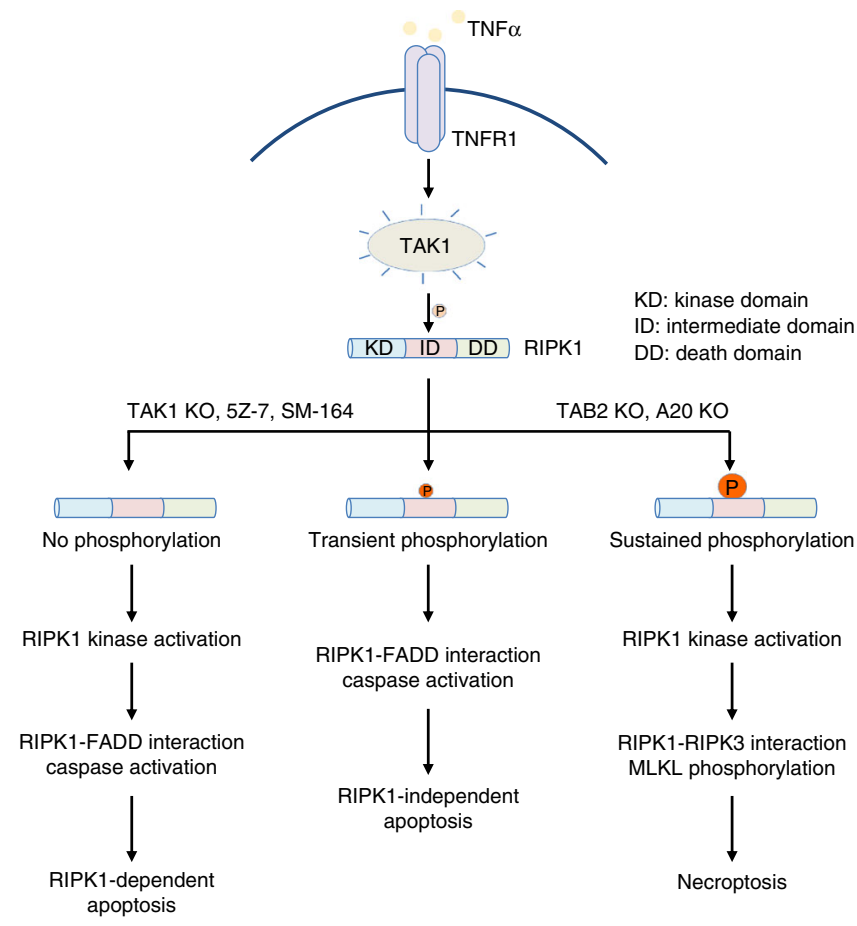

Fig. 8 TAK1-mediated RIPK1 phosphorylation dictates the activation multiple cell death pathways. Upon TNF $\alpha$ treatment, TAK1 is activated to mediate RIPK1 phosphorylation on the intermediate domain. Under normal conditions, TNF $\alpha / C H X$ induces transient phosphorylation of RIPK1 S321 by TAK1 and leads to RIPK1-independent apoptosis. Dysregulation of RIPK1 phosphorylation by TAK1 promotes its activation. When the phosphorylation of RIPK1 intermediate domain is blocked as in TAK1 KO cells, with inhibitor of TAK1 or IAP antagonist (SM-164), TNF $\alpha$ alone or $\mathrm{TNF} \alpha / \mathrm{CHX}$ treatment promotes the activation of RIPK1 and its binding with FADD to mediate RDA. On the other hand, hyperphosphorylation of RIPK1 intermediate domain, as in TNF $\alpha$-stimulated TAB2 KO and A2O KO cells, promotes its interaction with RIPK3 to mediate necroptosis

interaction but promotes the interaction of RIPK1 and RIPK3 to promote the activation of necroptosis in the absence of TNF $\alpha$ stimulation. Thus, absent, transient and sustained levels of TAK1mediated RIPK1 phosphorylation in TNF-RSC may represent three distinct states to dictate the execution of three alternative cell death mechanisms, RDA, RIPK1-independent cell death and necroptosis, by regulating the interaction of RIPK1 with FADD in kinase-dependent or -independent manner to mediate apoptosis, or with RIPK3 to mediate necroptosis (Fig. 8).

In TNF $\alpha$-stimulated cells, TAK1 is recruited to TNFR1 in a RIPK1-dependent manner to promote the phosphorylation of $\mathrm{I} \kappa \mathrm{B}$ kinase (IKK) and activation of NF- $\mathrm{KB}$ pathway, which has a powerful pro-survival role by inducing the expression of target genes that can block apoptosis, promote cell proliferation and stimulate inflammatory responses $6,28,29$. Our study defines a novel molecular contribution of TAK1 that controls the activity of RIPK1 kinase in mediating apoptosis, which is consistent, but distinct, from the role of TAK1 in promoting cell survival and inflammation in mediating NF- $\mathrm{KB}$ pathway activation. Supporting this idea, S321A mutation has no effect on the activation of $\mathrm{NF}-\kappa \mathrm{B}$ when cells are stimulated by TNF $\alpha$, as blocking NF- $\mathrm{\kappa B}$ activation by the addition of CHX, Smac mimetic, or TAK1 inhibitor, $5 Z-7$, is still required to significantly induce apoptosis in S321A(A/A) MEFs.

Our results demonstrate that deficiencies in TAB2 or A20 promote the phosphorylation of RIPK1 S321. Elevated levels of RIPK1 phosphorylation in the intermediate domain in TAB2 KO
MEFs drive the interaction of RIPK1 and RIPK3 to promote necroptosis. On the other hand, A20, encoded by the gene TNFAIP3, is an important ubiquitin-editing enzyme recruited to TNF-RSC to terminate multiple downstream events including NF-kB-mediated transcriptional response, RIPK1-mediated signaling and ultimately, the disassembly of the TNFR1 signaling comple $^{21,30}$. K63 ubiquitination of RIPK1 is abnormally elevated in A20-deficient cells ${ }^{31}$. Since K63 ubiquitination of TNFRSC is critical for mediating the activation of $\mathrm{TAK}^{4}$, the defective removal of K63 ubiquitination from TNF-RSC in A20deficient cells are predicted to lead to sustained TAK1 activation. However, since increased activation of TAK1 in A20-deficient cells is expected to promote sustained activation of NF- $\mathrm{kB}$, it has been puzzling as to why A20 deficiency also sensitizes cells to necroptosis ${ }^{7}$. Our study reveals a previously unexpected link between A20-regulated ubiquitination of TNF-RSC and TAK1mediated RIPK1 phosphorylation, which provides mechanistic insights as how A20 deficiency might promote the activation of RIPK1/RIPK3 complex to mediate necroptosis independent of $\mathrm{NF}-\kappa \mathrm{B}$ activation. Recent genome-wide association studies have identified single-nucleotide polymorphisms at the TNFAIP3/A20 locus in humans that are linked to susceptibility/resistance to inflammatory and autoimmune diseases ${ }^{32}$. Our study also suggests the possible clinical application of phospho-S320 in human RIPK1 (equivalent to S321 in murine RIPK1) as a biomarker for RIPK1-dependent cell death and inflammation.

\section{Methods}

Reagents antibodies and cell lines. The following commercial antibodies and reagents were used in this study: TAK1, Cell Signaling Technology (5206); RIPK1, Cell Signaling Technology (3493) and BD Biosciences (610459); TBK1, Cell Signaling Technology (3504); p-IKK $\alpha / \beta$, Cell Signaling Technology (2697); IKK $\beta$, Cell Signaling Technology (8943); I $\mathrm{B} \alpha$, Santa Cruz (sc-371); p-p38, Cell Signaling Technology (9211); p38, Cell Signaling Technology (9212); CYLD, Cell Signaling Technology (8462); FADD, Abcam (ab124812) and Santa Cruz (6036); $\alpha$-Tubulin Sigma-Aldrich (T9026); $\beta$-actin, Santa Cruz (81178). $\alpha$-Tubulin and $\beta$-actin antibodies were used with 5000 -fold dilution and other antibodies were used with 1000 -fold dilution. Uncropped scans of the most important blots were provided as Supplementary Fig. 7 in the Supplementary Information. 5Z-7 was from SigmaAldrich (O9890). Recombinant TAK1-TAB1 and GST-IKK $\alpha$ fusion proteins were obtained from Millipore (14-600) and Sigma (SRP5040). CellTiter-Glo luminescent cell viability kit was from Promega. $7-\mathrm{Cl}-\mathrm{O}-\mathrm{Nec}-1$ (Nec-1s) was made by custom synthesis. L929 and BV-2 cells were purchased from ATCC. TRAF2 and TRADD KO MEFs were provided by Dr. Zhenggang Liu. cIAP1/2 DKO MEFs were provided by Dr. John Silke. TAB2 KO MEFs were provided by Dr. Jun NinomiyaTsuji. Cell lines used in this study were tested every 3 months for mycoplasma contamination by MycoAlert Mycoplasma Detection Kit from Lonza.

Generation of anti-p-RIPK1 S321 and S332/334 antibodies. The phosphopeptides, VLQRMFpSLQHDC (S321) and CVPLPPpSRpSNSEQPG (S332/334), were synthesized and coupled to KLH carrier protein via Cys at the $\mathrm{C}$ terminus. Polyclonal anti-p-S321 or p-S332/334 RIPK1 antibodies were produced in rabbits against p-peptide antigen by ProteinTech.

AAV-RIPK1 vector and virus production. rAAV vector plasmids carrying the vector genomes with WT, S321A or S321E RIPK1 gene expression cassettes under the control of human thyroxine binding globulin (TBG) promoter, which directs efficient and sustaining transgene expression in liver-specific pattern ${ }^{24}$, are transfected individually into HEK293 cells with a packaging plasmid and adenovirus helper plasmid. The recombinant viruses were purified by standard $\mathrm{CsCl}$ gradient sedimentation method and desalted by dialysis ${ }^{33}$. The quality of vectors was tested by qPCR titration for DNase resistant vector genome concentration, silver-stained SDS-polyacrylamide gel analysis to establish the purity of each lot, electron microscopic analysis to independently test full/empty virus ratios ${ }^{33}$.

Generation of TAK1 ${ }^{\mathbf{F} / \mathbf{F}}$ and RIPK1 mutant MEFs. Super-ovulated female B6D2F1 mice (7-8 weeks old) were mated to B6D2F1 vasectomy males, and zygotes were collected from oviducts. Cas 9 mRNA and sgRNA were prepared by in vitro transcription. Single-stranded oligonucleotides (ssODN) were synthesized by Sangon. $100 \mathrm{ng} / \mu \mathrm{l}$ Cas 9 mRNA, $50 \mathrm{ng} / \mu \mathrm{l} \mathrm{sgRNA}$ and $100 \mathrm{ng} / \mu \mathrm{l} \mathrm{ssODN}$ were mixed in M2 medium (Sigma) and injected into the cytoplasm of zygote using a microinjector (FemtoJet, Eppendorf). The injected zygotes were cultured in KSOM with amino acids at $37^{\circ} \mathrm{C}$ under $5 \% \mathrm{CO}_{2}$ in air until transplantation. Thereafter, 20-25 
injected embryos were transferred into oviducts of pseudopregnant ICR females at 0.5 d.p.c. The pups were identified by both enzyme digestion and DNA sequencing. The positive mice were mated with WT C57BL/6 mice to produce heterozygous S321A or E mutant progenies, which are then backcrossed with $\mathrm{C} 57 \mathrm{BL} / 6$ mice.

Target sequence:

5'-TTTGACCTGCTCGGAGGTAA-3'

S321A ssODN (the synonymous mutation were highlighted by bold and point mutations were highlighted by Italic):

$5^{\prime}$-cattacagaaagagtatccagatcaaagcccagtgctgcagagaatgtttGCATTGCAGCATGAC TGTGTACCCTTGCCGccgagcaggtcaaattcaggtaactcacctattcgttcatttgcatactc- $3^{\prime}$

S321E ssODN

$5^{\prime}$-cattacagaaagagtatccagatcaaagcccagtgctgcagagaatgttt GAATTGCAGCATGACTGT

GTACCCTTGCCGccgagcaggtcaaattcaggtaactcacctattcgttcatttgcatactc-3

The founder mice carrying S321A or S321E mutant were backcrossed with C57BL/6 mice to produce heterozygous mice. WT and homozygous S321A(A/A) or WT and homozygous S321E(E/E) embryonic fibroblast cells were isolated from littermates of E13.5 embryos of heterozygous crosses. All experiments on mice were conducted according to the protocols approved by the Harvard Medical School Animal Care Committee and Institutional Animal Care and Use Committee of the Interdisciplinary Research Center on Biology and Chemistry of Chinese Academy of Sciences.

TAK1 ${ }^{\mathrm{F} / \mathrm{F}}$ MEFs were immortalized spontaneously in culture and infected by virus for the expression of Cre recombinase to generate TAK1-deficient MEF. RIPK1 S321A and S321E MEFs were immortalized by viral infection and subsequent expression of SV-40 large $\mathrm{T}$ antigen.

Caspase activity assay. Cell lysates were collected and mixed with substrate for caspase-8 (caspase-Glo 8 Assay, Promega) and the activity was determined according to the manufacturer's instructions.

In vitro caspase-8 cleavage assay. RIPK1 WT, S321A and S321E plasmids were transcribed and translated in vitro with $\left[{ }^{35} \mathrm{~S}\right]$-methionine labeling using TNT T7 Quick coupled transcription/translation kit (Promega). In vitro cleavage assay was performed in cleavage buffer containing $20 \mathrm{mM}$ HEPES ( $\mathrm{pH}=7.4$ ), $100 \mathrm{mM} \mathrm{NaCl}$, $20 \mathrm{mM}$ dithiothreitol and $0.5 \% \mathrm{NP}-40$ at $37^{\circ} \mathrm{C}$ for $1 \mathrm{~h}^{34}$.

In vitro kinase assay. Kinase-dead FLAG-RIPK1 variants with or without additional mutations or kinase-dead IKK $\beta$ were transiently expressed in $293 \mathrm{~T}$ and immunoprecipitated with anti-FLAG M2-agarose beads. Protein bound FLAG beads were incubated with $100 \mathrm{ng}$ recombinant TAK1 in kinase buffer containing $20 \mathrm{mM}$ HEPES $(\mathrm{pH}=7.3), 10 \mathrm{mM} \mathrm{MgCl} 2,10 \mathrm{mM} \mathrm{MnCl}_{2}, 10 \mu \mathrm{M}$ cold ATP and 1 $\mu \mathrm{Ci}$ of $\left[\gamma^{-}{ }^{32} \mathrm{P}\right] \mathrm{ATP}^{11}$. When indicated in the text, $1 \mu \mathrm{M} 5 \mathrm{Z}-7$ was added to the reaction mixture. The reactions were carried out at $30^{\circ} \mathrm{C}$ for $60 \mathrm{~min}$ and stopped by boiling in SDS-PAGE loading buffer at $95^{\circ} \mathrm{C}$ for $5 \mathrm{~min}$. Similar protocol was used for RIPK1 S321A phosphorylation assay. To confirm anti-p-S321-RIPK1 antibody specificity, 293T cells transiently expressing FLAG-RIPK1 WT or S321A were treated with $5 \mathrm{Z}-7(0.5 \mu \mathrm{M})$ and Nec-1s $(10 \mu \mathrm{M})$ to minimize RIPK1 phosphorylation background. RIPK1 WT and S321A mutant were purified and incubate with recombinant TAK1 or IKK $\alpha$ in kinase buffer without $\left[\gamma_{-}{ }^{32} \mathrm{P}\right] \mathrm{ATP}$ at $30^{\circ} \mathrm{C}$ for $30 \mathrm{~min}$.

Statistics. Data are expressed as the mean \pm s.e.m. Error bar, s.e.m. Pairwise comparisons between two groups were performed using the Student's $t$-test. Differences were considered statistically significant if $P<0.05\left(^{*}\right), P<0.01\left(^{* *}\right)$, $P<0.001\left({ }^{* *}\right)$ or not significant (n.s.). At least three independent biological repeats were included in each data point. Each experiment was repeated at least three times.

Data availability. The authors declare that the data supporting the findings of this study are available within the paper and its Supplementary Information Files.

Received: 13 October 2016 Accepted: 27 June 2017

Published online: 25 August 2017

\section{References}

1. Ofengeim, D. \& Yuan, J. Regulation of RIP1 kinase signalling at the crossroads of inflammation and cell death. Nat. Rev. Mol. Cell Biol. 14, 727-736 (2013).

2. Wallach, D., Kang, T. B., Dillon, C. P. \& Green, D. R. Programmed necrosis in inflammation: toward identification of the effector molecules. Science 352, aaf2154 (2016).

3. Kanayama, A. et al. TAB2 and TAB3 activate the NF-kappaB pathway through binding to polyubiquitin chains. Mol. Cell 15, 535-548 (2004).
4. Wang, C. et al. TAK1 is a ubiquitin-dependent kinase of MKK and IKK. Nature 412, 346-351 (2001).

5. Dondelinger, Y. et al. NF-kappaB-independent role of IKKalpha/IKKbeta in preventing RIPK1 kinase-dependent apoptotic and necroptotic cell death during TNF signaling. Mol. Cell 60, 63-76 (2015).

6. Mihaly, S. R., Ninomiya-Tsuji, J. \& Morioka, S. TAK1 control of cell death. Cell. Death Differ. 21, 1667-1676 (2014).

7. Onizawa, M. et al. The ubiquitin-modifying enzyme A20 restricts ubiquitination of the kinase RIPK3 and protects cells from necroptosis. Nat. Immunol. 16, 618-627 (2015).

8. Morioka, S. et al. TAK1 kinase switches cell fate from apoptosis to necrosis following TNF stimulation. J. Cell Biol. 204, 607-623 (2014).

9. Zhou, W. \& Yuan, J. Necroptosis in health and diseases. Semin. Cell Dev. Biol. 35, 14-23 (2014)

10. Degterev, A. et al. Chemical inhibitor of nonapoptotic cell death with therapeutic potential for ischemic brain injury. Nat. Chem. Biol. 1, 112-119 (2005)

11. Degterev, A. et al. Identification of RIP1 kinase as a specific cellular target of necrostatins. Nat. Chem. Biol. 4, 313-321 (2008).

12. Huttlin, E. L. et al. A tissue-specific atlas of mouse protein phosphorylation and expression. Cell 143, 1174-1189 (2010)

13. Zhang, J., Clark, K., Lawrence, T., Peggie, M. W. \& Cohen, P. An unexpected twist to the activation of IKKbeta: TAK1 primes IKKbeta for activation by autophosphorylation. Biochem. J. 461, 531-537 (2014).

14. Sato, S. et al. Essential function for the kinase TAK1 in innate and adaptive immune responses. Nat. Immunol. 6, 1087-1095 (2005).

15. Moriguchi, T. et al. A novel kinase cascade mediated by mitogen-activated protein kinase kinase 6 and MKK3. J. Biol. Chem. 271, 13675-13679 (1996).

16. Broglie, P., Matsumoto, K., Akira, S., Brautigan, D. L. \& Ninomiya-Tsuji, J. Transforming growth factor beta-activated kinase 1 (TAK1) kinase adaptor, TAK1-binding protein 2, plays dual roles in TAK1 signaling by recruiting both an activator and an inhibitor of TAK1 kinase in tumor necrosis factor signaling pathway. J. Biol. Chem. 285, 2333-2339 (2010).

17. Delhase, M., Hayakawa, M., Chen, Y. \& Karin, M. Positive and negative regulation of IkappaB kinase activity through IKKbeta subunit phosphorylation. Science 284, 309-313 (1999).

18. Ea, C. K., Deng, L., Xia, Z. P., Pineda, G. \& Chen, Z. J. Activation of IKK by TNFalpha requires site-specific ubiquitination of RIP1 and polyubiquitin binding by NEMO. Mol. Cell 22, 245-257 (2006).

19. Lu, J. et al. SM-164: a novel, bivalent Smac mimetic that induces apoptosis and tumor regression by concurrent removal of the blockade of cIAP-1/2 and XIAP. Cancer Res. 68, 9384-9393 (2008).

20. Pobezinskaya, Y. L. et al. The function of TRADD in signaling through tumor necrosis factor receptor 1 and TRIF-dependent Toll-like receptors. Nat. Immunol. 9, 1047-1054 (2008).

21. Wertz, I. E. et al. De-ubiquitination and ubiquitin ligase domains of A20 downregulate NF-kappaB signalling. Nature. 430, 694-699 (2004).

22. Newton, K. et al. RIPK3 deficiency or catalytically inactive RIPK1 provides greater benefit than MLKL deficiency in mouse models of inflammation and tissue injury. Cell Death Differ. 23, 1565-1576 (2016)

23. Gao, K. et al. Empty virions in AAV8 vector preparations reduce transduction efficiency and may cause total viral particle dose-limiting side-effects. Mol. Ther. Methods Clin. Dev. 1, 20139 (2014).

24. Yan, Z., Yan, H. \& Ou, H. Human thyroxine binding globulin (TBG) promoter directs efficient and sustaining transgene expression in liver-specific pattern. Gene 506, 289-294 (2012).

25. Lin, Y., Devin, A., Rodriguez, Y. \& Liu, Z. G. Cleavage of the death domain kinase RIP by caspase- 8 prompts TNF-induced apoptosis. Genes Dev. 13, 2514-2526 (1999).

26. Ofengeim, D. et al. Activation of necroptosis in multiple sclerosis. Cell Rep. 10 1836-1849 (2015).

27. Berger, S. B. et al. Cutting edge: RIP1 kinase activity is dispensable for normal development but is a key regulator of inflammation in SHARPIN-deficient mice. J. Immunol. 192, 5476-5480 (2014).

28. Blonska, M. et al. TAK1 is recruited to the tumor necrosis factor-alpha (TNFalpha) receptor 1 complex in a receptor-interacting protein (RIP)-dependent manner and cooperates with MEKK3 leading to NF-kappaB activation. J. Biol. Chem. 280, 43056-43063 (2005).

29. Hayden, M. S. \& Ghosh, S. Shared principles in NF-kappaB signaling. Cell 132 344-362 (2008).

30. Song, H. Y., Rothe, M. \& Goeddel, D. V. The tumor necrosis factor-inducible zinc finger protein A20 interacts with TRAF1/TRAF2 and inhibits NF-kappaB activation. Proc. Natl Acad. Sci. USA 93, 6721-6725 (1996).

31. Wertz, I. E. et al. Phosphorylation and linear ubiquitin direct A20 inhibition of inflammation. Nature 528, 370-375 (2015).

32. Mele, A., Cervantes, J. R., Chien, V., Friedman, D. \& Ferran, C. Single nucleotide polymorphisms at the TNFAIP3/A20 locus and susceptibility/ resistance to inflammatory and autoimmune diseases. Adv. Exp. Med. Biol. 809 163-183 (2014) 
33. Mueller, C., Ratner, D., Zhong, L., Esteves-Sena, M. \& Gao, G. Production and discovery of novel recombinant adeno-associated viral vectors. Curr. Protoc. Microbiol. 26, 14D.1.1-14D.1.21 (2012).

34. Cryns, V. L., Bergeron, L., Zhu, H., Li, H. \& Yuan, J. Specific cleavage of alphafodrin during Fas- and tumor necrosis factor-induced apoptosis is mediated by an interleukin-1beta-converting enzyme/Ced-3 protease distinct from the poly (ADP-ribose) polymerase protease. J. Biol. Chem. 271, 31277-31282 (1996).

\section{Acknowledgements}

We thank Dr. Zhenggang Liu for sharing TRADD KO and TRAF2 KO MEFs, Dr. John Silke for cIAP1/2 DKO MEFs and Dr. Jun Ninomiya-Tsuji for TAB2 KO MEFs. We thank Dr. Slawomir Dziedzic for critical reading of this manuscript. This work was supported in part by grants from the National Institute on Aging (US) (1R01AG047231), the NINDS (US) (1R01NS082257), the grants from the Chinese Academy of Sciences, the National Key R\&D Program of China, the China Ministry of Science and Technology Program (2014ZX09102001-002) and the China National Natural Science Foundation (31530041) and the Chinese Academy of Sciences and the National Science Foundation in China (to J.Y.), and from Shanghai Yangfan Plan for the Young Scientific Talents (15YF1414700) (to L.S.). Y.I. was supported in part by a Postdoctoral Fellowship from Daiichi Sankyo Foundation of Life Science, the Nakatomi Foundation, Mochida Memorial Foundation for Medical and Pharmaceutical Research and the Japan Society for the Promotion of Science (No. 25•803).

\section{Author contributions}

J.G. performed majority of the experiments. Y.I. and P.A. identified 5Z-7 inhibition on RIPK1 S321 phosphorylation. L.S. generated the RIPK1 S321A(A/A) and S321E(E/E) knock-in MEFs. J.C. characterized RIPK1 S321 phosphorylation in primary cells and TBK1 KO cells. A.K.M. and H.Z. performed the animal AAV experiments. A.T.O. constructed RIPK1 EEEE plasmid. D.X. generated TBK1 KO MEF and BV-2 cells. B.S. and A.N. did mass spec analysis. G.G. generated AAVs. S.A. provided the $\mathrm{TAK1}^{\mathrm{F} / \mathrm{F}}$ mice.
J.G. and J.Y. conceived and designed the experiments, analyzed the data and wrote the manuscript. J.Y. directed and coordinated all of the experiments. All authors discussed the results and reviewed the manuscript.

\section{Additional information}

Supplementary Information accompanies this paper at doi:10.1038/s41467-017-00406-w.

Competing interests: The authors declare no competing financial interests.

Reprints and permission information is available online at http://npg.nature.com/ reprintsandpermissions/

Publisher's note: Springer Nature remains neutral with regard to jurisdictional claims in published maps and institutional affiliations.

(c) (i) Open Access This article is licensed under a Creative Commons Attribution 4.0 International License, which permits use, sharing, adaptation, distribution and reproduction in any medium or format, as long as you give appropriate credit to the original author(s) and the source, provide a link to the Creative Commons license, and indicate if changes were made. The images or other third party material in this article are included in the article's Creative Commons license, unless indicated otherwise in a credit line to the material. If material is not included in the article's Creative Commons license and your intended use is not permitted by statutory regulation or exceeds the permitted use, you will need to obtain permission directly from the copyright holder. To view a copy of this license, visit http://creativecommons.org/ licenses/by/4.0/.

(C) The Author(s) 2017 